\title{
Two Practical Strategies for Developing Resultant Muscle Torque Production Using Elastic Resistance Device
}

S.J. Aboodarda, A. Yusof, N.A. Abu Osman, and F. Ibrahim

INTRODUCTION

Elastic resistance exercise (ER) is well established as an

effective mode of training in rehabilitation and fitness

setting $[1,2]$. Numerous studies have evaluated the clinical

features of utilizing ER and recommended it for patients and

older adults to strength and pain impairment, balance and

proprioception enhancement, increase range of motion after

trauma [3-7]. Furthermore, few investigators have suggested

ER for prevention of injuries and developing muscle

strength rather than curing, particularly among healthy

individuals [8]. However, there is controversial evidence

regarding application of ER for improving muscle strength

among healthy individuals [9]. Reportedly, ER cannot lead

muscle to its maximal activation level due to providing

inadequate external force $[4,7]$. Based on this viewpoint,

utilizing ER has been confined to the initial stages of

rehabilitation protocols $[1,3]$. On this basis, in the current

study two strategies are proposed to enhance the magnitude

of elastic force. Firstly, $30 \%$ of the initial length of the

elastic material are reduced [9, 10]. Secondly, additional

elastic bands are utilized in parallel to the current unit [7].

The aim of the first and the second strategies were to

enhance the provided force by ER device at the beginning

of lifting motion and throughout the whole range of motion

(ROM), respectively.

We hypothesize that applying these strategies can improve muscle torque production during intensive knee extension exercises. The purpose of this study, therefore, is to quantify and compare Resultant Muscle Torque (RMT) 
production within performing 8-RM seated knee extension in contribution of two types of ER training.

MAteRIALS AND METHOD

Experimental Approach to the Problem

All data were collected within one testing session. Subjects completed 8-RM seated knee extension by elastic tubing with original elongation (E0) and elastic tubing with $30 \%$ decrement of original elongation (E30). The external force, linear acceleration and range of motion data were collected and synchronized using a 16 bit acquisition mode with an eight channel TeleMyo ${ }^{\text {TM }}$ 2400T G2 (Noraxon, Scottsdale, Arizona, USA) system.

Subjects

Seven female (mean, SD; $22.4 \pm 4.7$ year, $60.05 \pm 6.17$

$\mathrm{kg}, 158 \pm 3 \mathrm{~cm})$ and 9 male $(24.0 \pm 3.6$ year, $78.14 \pm 7.2 \mathrm{~kg}$, $174 \pm 7 \mathrm{~cm}$ ) healthy volunteers were recruited for this study. None of the subjects had experience of participating in any resistance training program in the past 12 months. This study was approved by ethic committee Sports Center, University Malaya and all participants signed informed consent forms.

\section{Instrumentation}

Range of motion in dominant knee was monitored using a 2D electrogoniometer in $200 \mathrm{uv}$ and a two dimension accelerometer $(10 \mathrm{~g})$ was places at the lateral side of the shank to measure linear acceleration of the shank and foot

(Noraxon, Scottsdale, Arizona, USA). Data acquisition package Myoresearch-XP, Master Edition (Noraxon, Scottsdale, Arizona, USA) was used to collect data from all 8-RM while the first, 5 th and 8 th repetitions has been selected for data analyze. Various color code of Thera-Band elastic tubing (Hygienic Corporation, Akron, $\mathrm{OH}$ ) were used to provide elastic resistance. In order to avoid any biomechanical differentiation in position of the subjects, pattern of the movement and range of motion, the nautilus 
machine chair (Nautilus, Vancouver, WA) was used for elastic resistances as well. The original length of elastic tubing was measured from axis of the elastic tubing anchored to the base of the nautilus machine (underneath the seat) and a load cell (Noraxon, Scottsdale, Arizona, USA) which was connected to a custom made leather shin pat of elastic device.

\section{Experimental Measurement}

All data were collected within one testing session and the order of the measurement was randomized across two exercise modalities. Following warm up, the electrogoniometer and accelerometer were strapped to the subject`s dominant knee and ankle respectively. Then, subjects were seated on the leg extension nautilus machine chair. They completed 8-RM tests with 2 modes of resistance exercise within $80^{\circ}$ to $180^{\circ}$ of knee extension. Lifting more than 8 repetitions with the current resistance in the assigned range of motion required the subject to execute the next trial with heavier weight or undertaking elastic resistance with next color code of elastic tubing or add up a tubing until the subject was no longer able to perform more than 8 repetitions. The first, the 5 th and the 8 th repetitions were divided into concentric and eccentric segments and value of every segment was divided in to 3 equal phases (totally 6 phases: 3 concentric and 3 eccentric). The acceleration (g) and the average of external force $(\mathrm{N})$ were calculated for each phase of movement.

Full text is available at :

http://link.springer.com/chapter/10.1007/978-3-642-21729-6 64 